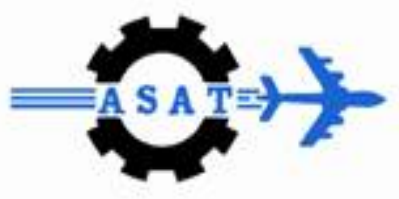

\title{
Aerodynamic Design of a Propeller Applied for the Aircraft ZLIN-226
}

\author{
Daniel Svrcek*, Vaclav Broz $^{* *}$
}

\begin{abstract}
The aerodynamic design of an aircraft propeller requires a proper choice of design conditions among which the engine characteristics (power, revolutions) and the design flight speed are essential. Especially fixed propellers are considerably affected by the design point because their best operation corresponds to a single flight speed only.

The paper deals with the design of aerodynamic characteristics of a propeller aimed at achieving maximum efficiency at a chosen design regime. Frequently the design point is selected at the flight speed providing the maximum rate of climb. The presented propeller design is specified for the Z-226 airplane with the WALTER M-137 engine.
\end{abstract}

Keywords: Propellers, Aerodynamic design

\begin{tabular}{lll}
\multicolumn{2}{l}{ Nomenclature } & \\
$C$ & {$[-]$} & Correction factor (effect of number of blades) \\
$D$ & {$[m]$} & Propeller diameter \\
$Q$ & {$[N]$} & Drag \\
$L$ & {$[N]$} & Lift \\
$N$ & {$[N]$} & Circumferential force (plane of rotation) \\
$P$ & {$[W]$} & Power \\
$R$ & {$[m]$} & Propeller radius \\
$S$ & {$\left[m^{2}\right]$} & Propeller disc area \\
$T$ & {$[N]$} & Thrust \\
$\beta_{0}$ & {$[0]$} & Flow angle \\
$\beta_{1}$ & {$[0]$} & Flow angle (induced velocity included) $w_{1}$ \\
$\Gamma$ & {$\left[m^{2} \cdot s^{-1}\right]$} & Circulation
\end{tabular}

\footnotetext{
* Senior lecturer, Ing. Daniel Švrček, PhD., Slovak University of Technology in Bratislava, Faculty of Materials Science and Technology in Trnava, Paulinska 16, 91724 Trnava, Slovak Republic, Tel.: +421335511601, e-mail: agrolet@stonline.sk

** professor, Václav Brož, Prof. Dr. Ing. Department of Aerospace Engineering, Faculty of Mechanical Engineering, Czech Technical University in Prague, Karlovo nám. 13, 12135 Prague 2, Tel: +420 22 4357422, e-mail:brozv@fsik.cvut.cz
} 


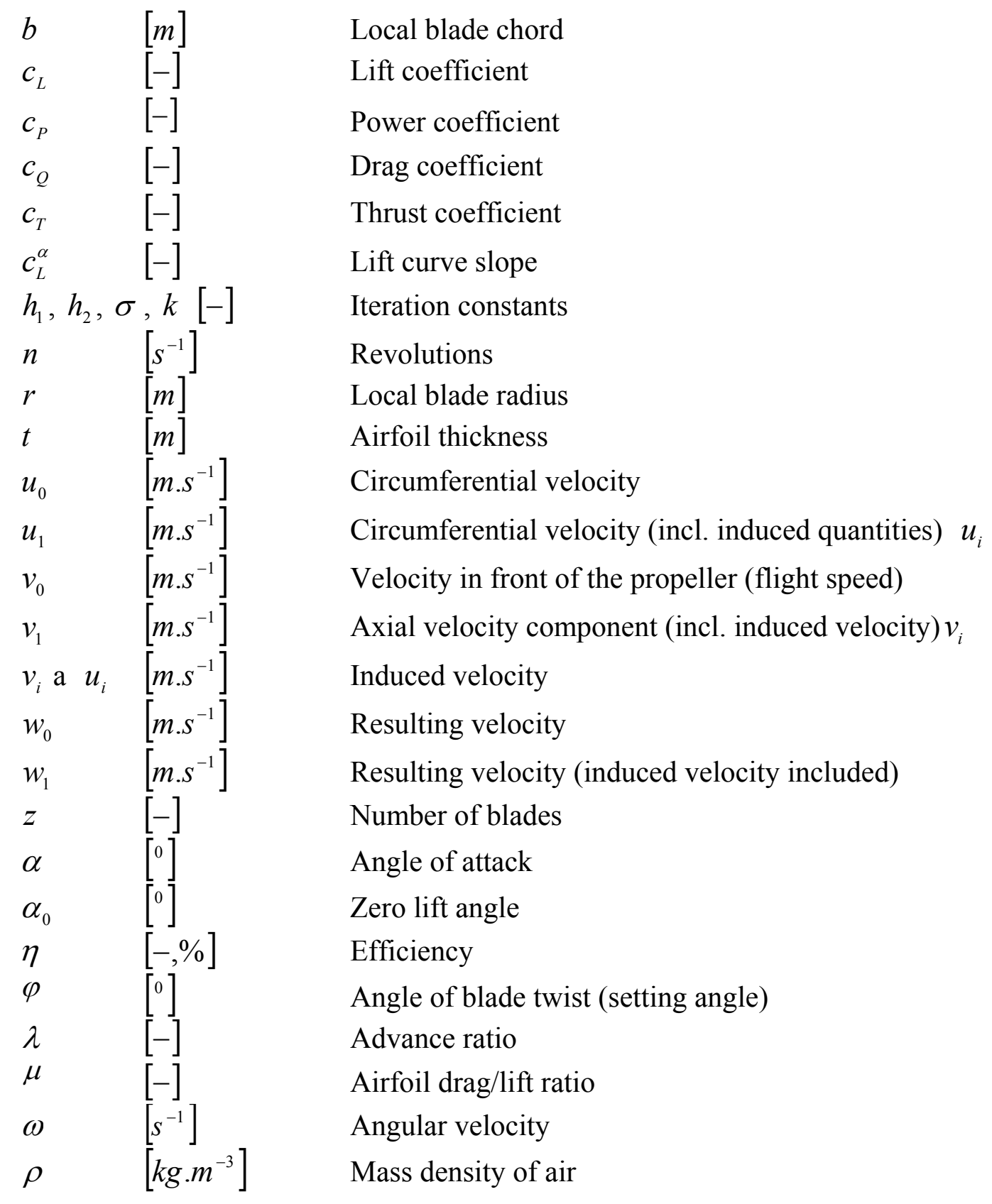

\section{Introduction}

The purpose of a propeller is to achieve maximum thrust at the given engine parameters (power, r.p.m.) and selected flight parameters (flight speed, altitude). By considering velocity and force relations on a propeller blade it is possible to establish the mathematical description in a form of propeller equations which analyzed lead to the conditions for the optimum distribution of circulation along the propeller blade. The magnitude of circulation has a direct link to propeller geometric and aerodynamic parameters satisfying the selected design regime.

The paper focuses on the aerodynamic design of a propeller blade giving maximum efficiency at the particular advance ratio. A set of equations has been derived the solution of which leads to optimum distribution of circulation in relation to the power coefficient, advance ratio and number of blades. 


\section{Design Specification}

The initial parameters for the design of a propeller include engine parameters (power, revolutions), propeller diameter and flight speed. In describing aerodynamic properties of propellers essential quantities are given by the relations:

Propeller thrust

$T=\rho n^{2} D^{4} c_{T}$

Power

$P=\rho n^{3} D^{5} c_{P}$

Advance ratio

$\lambda=\frac{v_{0}}{n \cdot D}$

The propeller efficiency comes from the previous relations as

$\eta=\frac{T v_{0}}{P} \quad$ then $\quad \eta=\lambda \frac{c_{T}}{c_{P}}$.

The task to achieve maximum efficiency at the fixed values of the power coefficient and advance ratio leads to the condition of achieving $c_{T} \rightarrow \max [2]$.

\section{Set of Propeller Equations}

In the following relations the indices 0,1 describe the flow in front of a propeller (index , , ") and in the propeller disc (index ," "), as indicated in Fig.1. The relation between the local lift values and the total lift can be expressed as

$$
\begin{aligned}
& L=\frac{1}{2} \rho w_{1}^{2} c_{L} S=\frac{1}{2} \rho \int_{0}^{R} w_{1(r)}^{2} c_{L(r)} b_{(r)} d r \\
& d L=\frac{1}{2} \rho w_{1}^{2} c_{L} b d r
\end{aligned}
$$

Introducing the concept of circulation and the Joukovski - Kutta theorem makes possible to write relations

$$
\begin{aligned}
& \Gamma=\frac{1}{2} \rho w_{1} c_{L} b \\
& d L=\rho w_{1} \Gamma d r
\end{aligned}
$$

These terms when inserted into (5) give the lift resultant which depends on the distribution of circulation along a propeller blade. The previous procedure transfers the task of gaining 
maximum efficiency to the problem of determining the optimum distribution of circulation. To facilitate the numerical procedures the non-dimensional quantities are introduced

$$
\bar{\Gamma}=\frac{\Gamma}{4 \pi R^{2} \omega} \quad \bar{b}=\frac{z b}{4 \pi R} \quad \bar{r}=\frac{r}{R} \quad \bar{v}_{1}=\frac{v_{1}}{R \omega} \quad \bar{u}_{1}=\frac{u_{1}}{R \omega}
$$

where the velocity components are indicated in Fig. 1.

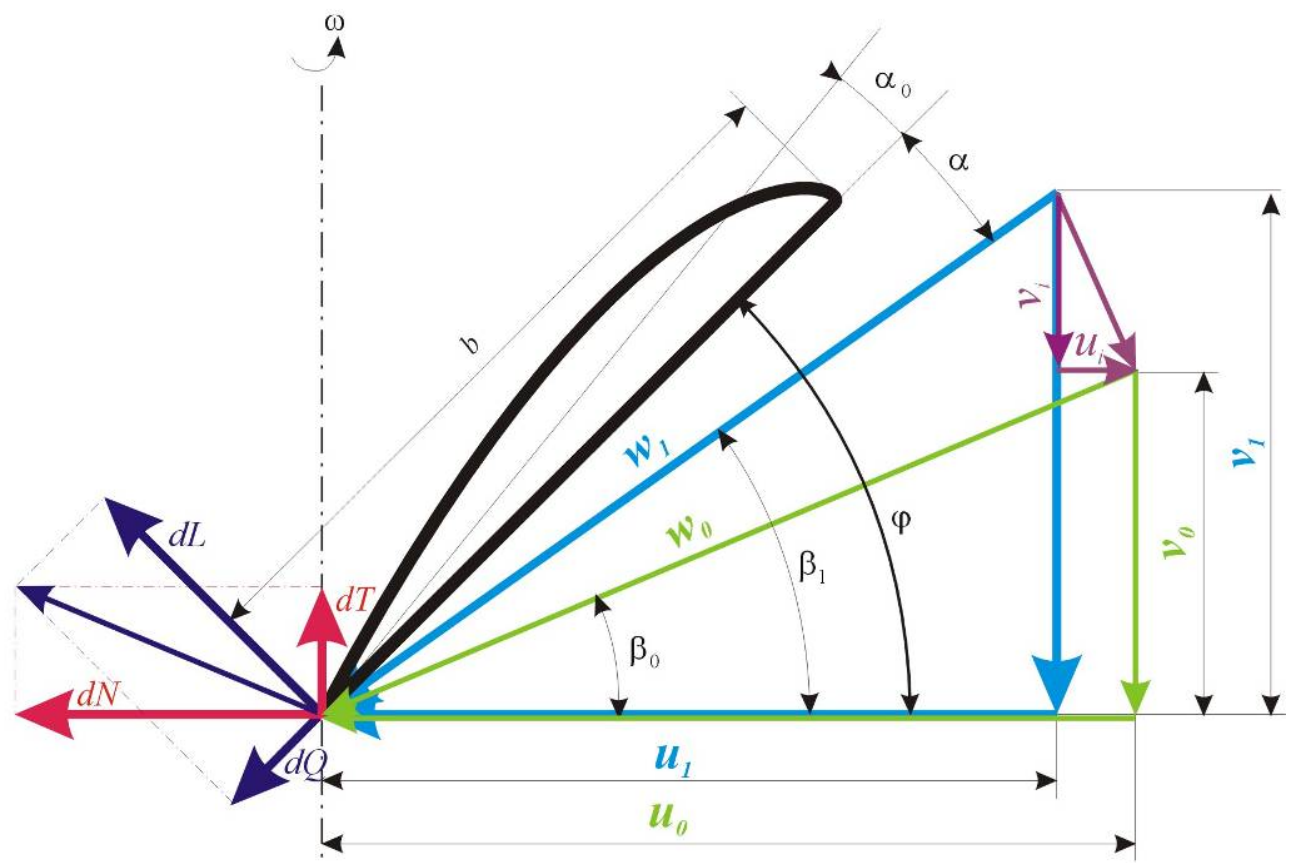

Fig. 1. Velocity and force components

\section{Velocity Relations at a Local Blade Section}

The resulting velocity at the local radius including induced effects is composed of axial and tangential components

$\bar{w}_{1}=\sqrt{\left(\bar{u}_{1}^{2}+\bar{v}_{1}^{2}\right)}$

$\bar{u}_{1}=\bar{u}_{0}-\bar{u}_{i}$

$\bar{v}_{1}=\bar{v}_{0}+\bar{v}_{i}$

or by means of $(12)$

$\bar{u}_{1}=\bar{r}-\bar{u}_{i}$

$\bar{v}_{1}=\frac{\lambda}{. \pi}+\bar{v}_{i}$ 
The simplest vortex system of a propeller is represented by a system of concentric vortex cylinders with a continuous distribution of circulation (by Joukovski) which gives the following relations for velocity components as functions of circulation

$$
\begin{aligned}
& \bar{u}_{1}=\bar{r}-\frac{\bar{\Gamma}}{\bar{r}} \\
& \bar{v}_{1}=\frac{\lambda}{2 \pi}+\sqrt{\left(\left(\frac{\lambda}{2 \pi}\right)^{2}+\bar{\Gamma}(1+\bar{\Gamma})\right)} .
\end{aligned}
$$

The vortex system of question does not fully conform to experimental results because it strictly corresponds to the infinite number of blades. As a consequence the error increases with the advance ratio, the propeller power loading and the decrease in number of blades.

It is recommended to improve the error by a correction factor depending on the advance ratio and number of blades [1]

$$
C=1+1.803\left(\frac{1}{z}\right)^{1,16} \lambda^{2}-0.459\left(\frac{1}{z}\right)^{1,062} \lambda^{3}+0.0243\left(\frac{1}{z}\right)^{0,835} \lambda^{4}
$$

Substituting into (15), (16), the velocity components become

$$
\bar{u}_{1}=\bar{r}-C \frac{\bar{\Gamma}}{\bar{r}}
$$

$$
\bar{v}_{1}=\frac{\lambda}{2 \pi}(2-C)+C \sqrt{\left(\left(\frac{\lambda}{2 \pi}\right)^{2}+\bar{\Gamma}(1+\bar{\Gamma})\right)}
$$

The following step is to specify links between kinematic and structural quantities by the geometric parameters: angle of blade twist, local sectional chord and airfoil thickness to conform the flow relations (Fig. 1)

$$
\begin{aligned}
& \beta_{1}=\operatorname{arctg} \frac{\bar{v}_{1}}{\bar{u}_{1}} \\
& \varphi=\beta_{1}+\alpha \\
& c_{L}=c_{L}^{\alpha}\left[\left(\varphi-\alpha_{0}\right)-\operatorname{arctg} \frac{\bar{v}_{1}}{\bar{u}_{1}}\right]
\end{aligned}
$$

By analyzing the previous relations, it is possible to get to the conclusion that the structural parameters $\varphi, b$ a $t$ of the propeller at the particular blade section are determined by the local value of circulation $\bar{\Gamma}$ [1]. 


\section{Distribution of Optimum Circulation}

Based on the previous considerations on velocity and geometric relations the task to calculate and design the propeller blade is simplified to the determination of optimum distribution of circulation.

The specific method to solve this problem and utilize its result for a real propeller design follows the procedures described in [4]. An iteration method was used where the successive steps lead to the final value of local circulation. The numerical process is terminated when the accuracy criterion for two consecutive steps is satisfied $\left|\bar{\Gamma}-\bar{\Gamma}_{(-1)}\right| \leq 1.10^{-4}$. Iteration constants

$$
h_{1}=\frac{\lambda}{2 \pi}(2-C)+C \frac{\left(\frac{\lambda}{2 \pi}\right)^{2}+\frac{3 \bar{\Gamma}_{(-1)}}{2}}{\sqrt{\left[\left(\frac{\lambda}{2 \pi}\right)^{2}+\bar{\Gamma}_{(-1)}\right]}} \quad h_{2}=C \frac{\left(\frac{\lambda}{2 \pi}\right)^{2}+\frac{3 \bar{\Gamma}_{(-1)}}{4}}{\sqrt{\left[\left(\frac{\lambda}{2 \pi}\right)^{2}+\bar{\Gamma}_{(-1)}\right]}} \quad \sigma=\frac{k \bar{r}-\mu}{1+k \mu \bar{r}}
$$

form auxiliary quantities for the final relation

$$
\bar{\Gamma}=\bar{r}\left(\frac{\bar{r}+\sigma\left(h_{1}-h_{2} \bar{\Gamma}_{(-1)}\right)}{2 C-\bar{r} \sigma h_{2}}\right) \text {. }
$$

The calculated values represent the optimum distribution of circulation directly giving the distribution of local chords and setting angles. Moreover it enables to calculate even the thrust and power coefficients [1].

\section{Demonstration of a Propeller Design}

Based on the procedure described in the paper a number of propellers were designed and more than 100 manufactured in the diameter extent of $D=0.15 \mathrm{~m}$ to $D=3.0 \mathrm{~m}$.

Example of the design for the Z-226 airplane equipped with the M-137 AK engine:

The design point - input data:

$D=2.0 \mathrm{~m}, \quad v_{0}=36.11 \mathrm{~m} \cdot \mathrm{s}^{-1}=130 \mathrm{~km} \cdot \mathrm{hod}^{-1}, n=44.16 \mathrm{~s}^{-1}=2650 \mathrm{~min}^{-1}, P=125 \mathrm{~kW}$

The input values determine the following parameters $\lambda=0.4088, c_{P}=0.0370, \mathrm{C}=1.120$ which enter the iterative process and lead to the distribution of optimum circulation $\bar{\Gamma}$ given in Fig. 2. Numerical values of circulation and velocity components are summarized in Table 1.

The next step consists in the calculation of the lift distribution $c_{L} \cdot \bar{b}$ from (7). The $c_{L} \cdot \bar{b}$ values in relation to the selected airfoil RAF 6 and its aerodynamic characteristics provide the resulting geometric parameters of the blade (Table 2 ), Fig.3 and Fig.4.

Calculation of the integral characteristics gives the following data

$c_{T}=0.06598, c_{P}=0.03773 \eta=71.48 \%, T=2522.76 \mathrm{~N}$ and $P=127.43 \mathrm{~kW}$. 


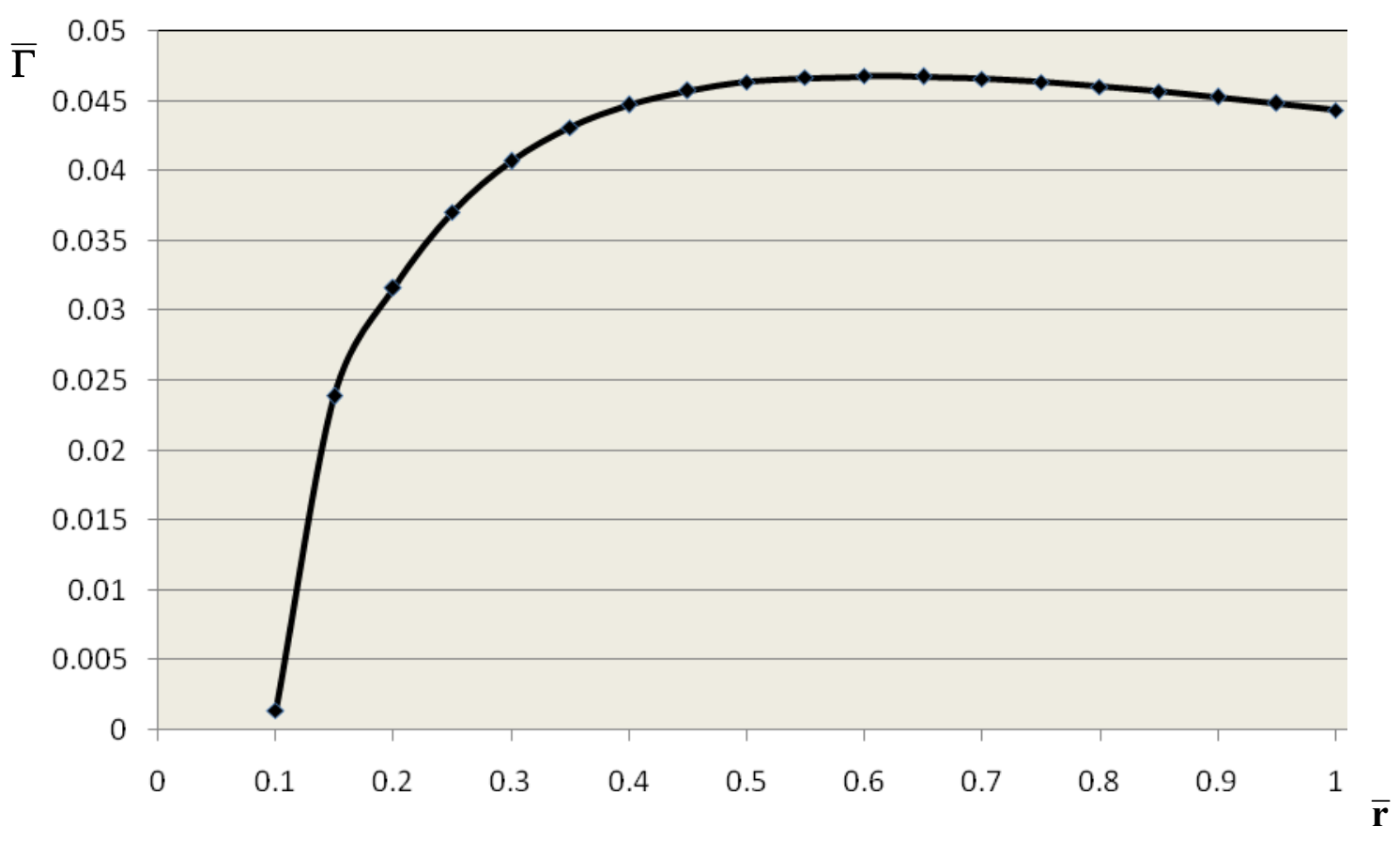

Fig. 2. Distribution of circulation $\bar{\Gamma}$ along the propeller blade

Table 1. Distribution of circulation and velocity components on the propeller blade

\begin{tabular}{|l|l|l|l|l|}
\hline \multicolumn{1}{|c|}{$\bar{r}$} & \multicolumn{1}{|c|}{$\bar{\Gamma}$} & \multicolumn{1}{c|}{$\bar{u}_{1}$} & \multicolumn{1}{c|}{$\bar{v}_{1}$} & \multicolumn{1}{c|}{$\bar{w}_{1}$} \\
\hline 0 & 0 & 0 & 0 & 0 \\
\hline 0.1 & 0.00138 & 0.08611 & 0.14004 & 0.16440 \\
\hline 0.15 & 0.02386 & 0.13408 & 0.14642 & 0.19854 \\
\hline 0.2 & 0.03161 & 0.18419 & 0.15105 & 0.23821 \\
\hline 0.25 & 0.03704 & 0.23518 & 0.15415 & 0.28120 \\
\hline 0.3 & 0.04070 & 0.28643 & 0.15618 & 0.32624 \\
\hline 0.35 & 0.04313 & 0.33767 & 0.15751 & 0.37260 \\
\hline 0.4 & 0.04472 & 0.38881 & 0.15836 & 0.41983 \\
\hline 0.45 & 0.04573 & 0.43983 & 0.15891 & 0.46766 \\
\hline 0.5 & 0.04635 & 0.49073 & 0.15923 & 0.51591 \\
\hline 0.55 & 0.04667 & 0.54151 & 0.15940 & 0.56448 \\
\hline 0.6 & 0.04679 & 0.59220 & 0.15947 & 0.61329 \\
\hline 0.65 & 0.04675 & 0.64280 & 0.15945 & 0.66228 \\
\hline 0.7 & 0.04660 & 0.69334 & 0.15937 & 0.71142 \\
\hline 0.75 & 0.04636 & 0.74381 & 0.15924 & 0.76067 \\
\hline 0.8 & 0.04605 & 0.79424 & 0.15907 & 0.81001 \\
\hline 0.85 & 0.04568 & 0.84462 & 015888 & 0.85943 \\
\hline 0.9 & 0.04528 & 0.89496 & 0.15866 & 0.90892 \\
\hline 0.95 & 0.04483 & 0.94528 & 0.15842 & 0.95846 \\
\hline 1 & 0.04436 & 0.99556 & 0.15817 & 1.00805 \\
\hline
\end{tabular}


Table 2. Final aerodynamic and geometric characteristics

\begin{tabular}{|l|l|l|l|l|l|l|l|}
\hline $\bar{r}$ & $c_{L} . \bar{b}$ & $c_{L}$ & $b[\mathrm{~m}]$ & $t[\%]$ & $\alpha[\mathrm{o}]$ & $\varphi\left[^{\mathrm{o}}\right]$ \\
\hline 0 & 0.0 & 0.0 & & & & \\
\hline 0.1 & 0.01689 & 0.5768 & & & & \\
\hline 0.15 & 0.02404 & 0.8346 & & & & \\
\hline 0.2 & 0.02654 & 0.9114 & & & & \\
\hline 0.25 & 0.02634 & 0.8644 & & & & \\
\hline 0.3 & 0.02495 & 0.8081 & 0.194 & 31.09 & 2.49 & 31.09 \\
\hline 0.35 & 0.02315 & 0.7737 & 0.188 & 27.01 & 2.00 & 27.01 \\
\hline 0.4 & 0.02130 & 0.7416 & 0.1805 & 23.57 & 1.40 & 23.57 \\
\hline 0.45 & 0.01956 & 0.7092 & 0.1733 & 20.92 & 1.06 & 20.92 \\
\hline 0.5 & 0.01796 & 0.6797 & 0.1661 & 18.90 & 0.92 & 18.90 \\
\hline 0.55 & 0.01653 & 0.6540 & 0.1589 & 17.31 & 0.90 & 17.31 \\
\hline 0.6 & 0.01526 & 0.6322 & 0.1517 & 16.07 & 1.00 & 16.07 \\
\hline 0.65 & 0.01412 & 0.6141 & 0.1445 & 15.08 & 1.14 & 15.08 \\
\hline 0.7 & 0.01310 & 0.5998 & 0.1372 & 14.14 & 1.20 & 14.14 \\
\hline 0.75 & 0.01219 & 0.5890 & 0.1300 & 13.38 & 1.29 & 13.38 \\
\hline 0.8 & 0.01137 & 0.5816 & 0.1228 & 12.72 & 1.39 & 12.72 \\
\hline 0.85 & 0.01063 & 0.5777 & 0.1156 & 12.21 & 1.56 & 12.21 \\
\hline 0.9 & 0.00996 & 0.5774 & 0.1084 & 11.81 & 1.76 & 11.81 \\
\hline 0.95 & 0.00935 & 0.5808 & 0.1012 & 11.56 & 2.05 & 11.56 \\
\hline 1 & 0.00880 & 0.5883 & 0.0940 & 11.30 & 2.27 & 11.30 \\
\hline
\end{tabular}

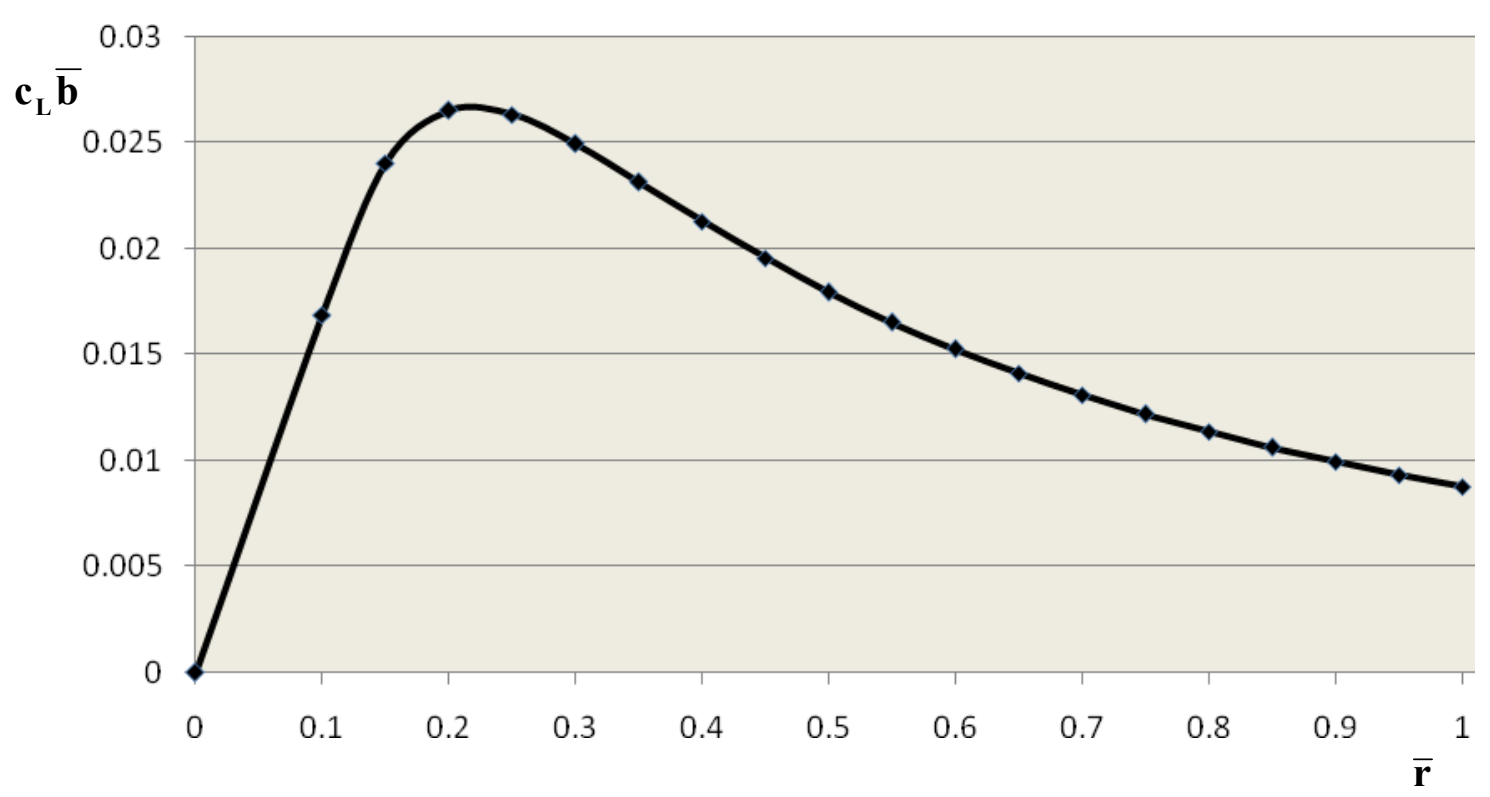

Fig. 3. Lift distribution $c_{L} \cdot \bar{b}$ 


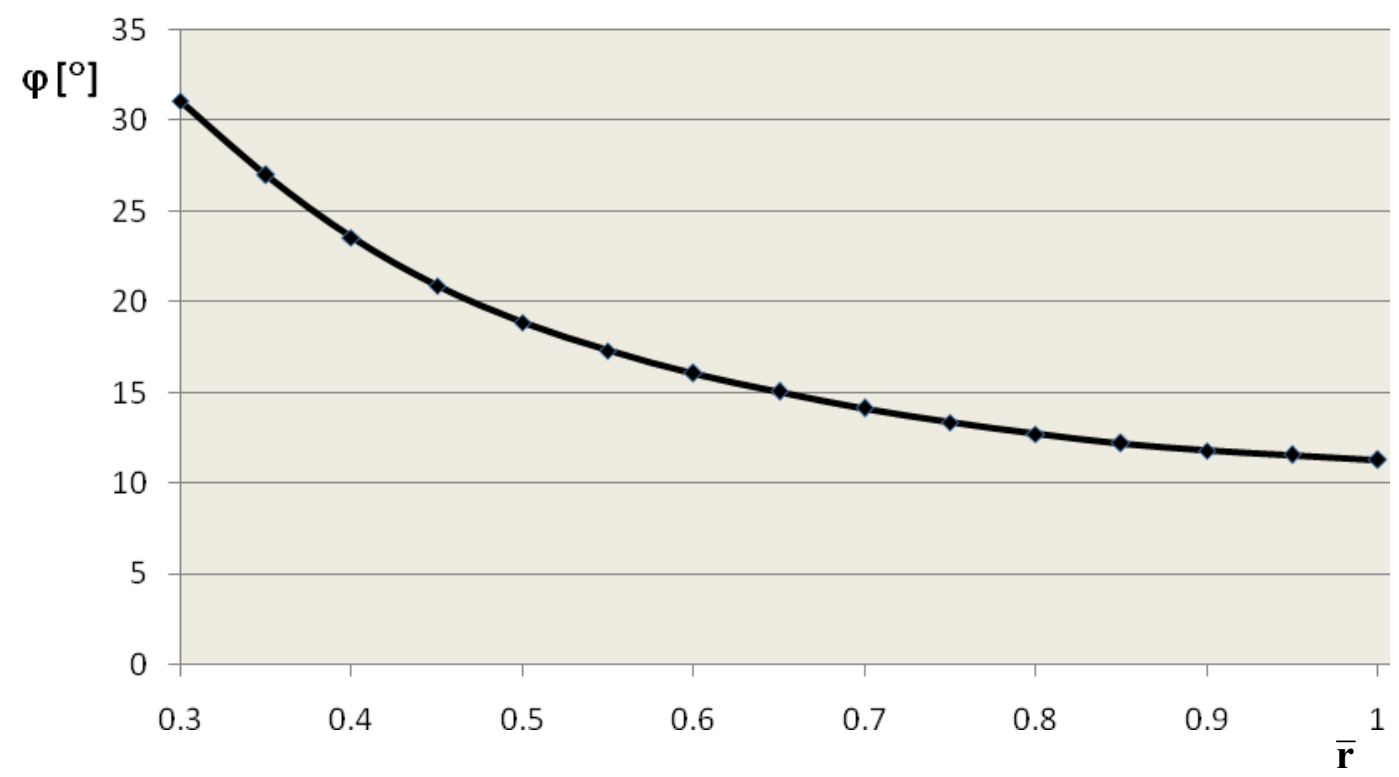

Fig. 4. Distribution of blade twist $\varphi\left[{ }^{\circ}\right]$

Eventually the propeller of question (SC-Z226) was manufactured and advanced to flight tests, Figs. 5 \& 6.

The rate of climb was selected as a primary criterion. The difference was $0.8-1.1 \mathrm{~m} . \mathrm{s}^{-1}$ and increased the rate of climb to $4.5 \mathrm{~m} . \mathrm{s}^{-1}$ in favor of the new design.

The thrust characteristics at low speeds also received better evaluation by the test pilots leading consequently to a shorter ground run and total take-off distance. The propeller efficiency was assessed as better in the full extent of flight speeds.

\section{Conclusion}

The presented method makes possible to design structural parameters of a propeller blade. It is based on the determination of optimum distribution of circulation leading to high propeller efficiency. The design parameters (propeller geometry) meet the specifications given by the combination of the engine parameters and selected flight regime

\section{References}

[1] BROŽ, V.: Distribution of Circulation on a Propeller Blade. Zpravodaj VZLÚ, Vol. 5, 1963, pp. 17-23 (in Czech)

[2] BROŽ, V.: Aerodynamic Design of a High Efficiency Propeller. Zpravodaj VZLÚ, Vol. 5, 1966, pp. 324-328 (in Czech)

[3] BROŽ, V., SLAVÍK, S.: Diagrams of Optimum Distributions of Circulation on a Propeller Blade. Zpráva VZLÚ No. V-1348/79, Praha, 1978 (in Czech)

[4] SLAVÍK, S.: Distribution of Circulation on a Propeller Blade including the Nonlinear Effect of the Number of Blades. Zpravodaj VZLÚ, Vol. 5, 1985, pp. 371-375 (in Czech)

[5] ŠVRČEK, D.: Propellers. Proceedings of the Conference on Manufacturing of Microlights. Bratislava, 1998, pp. 1-10. (in Slovak) 


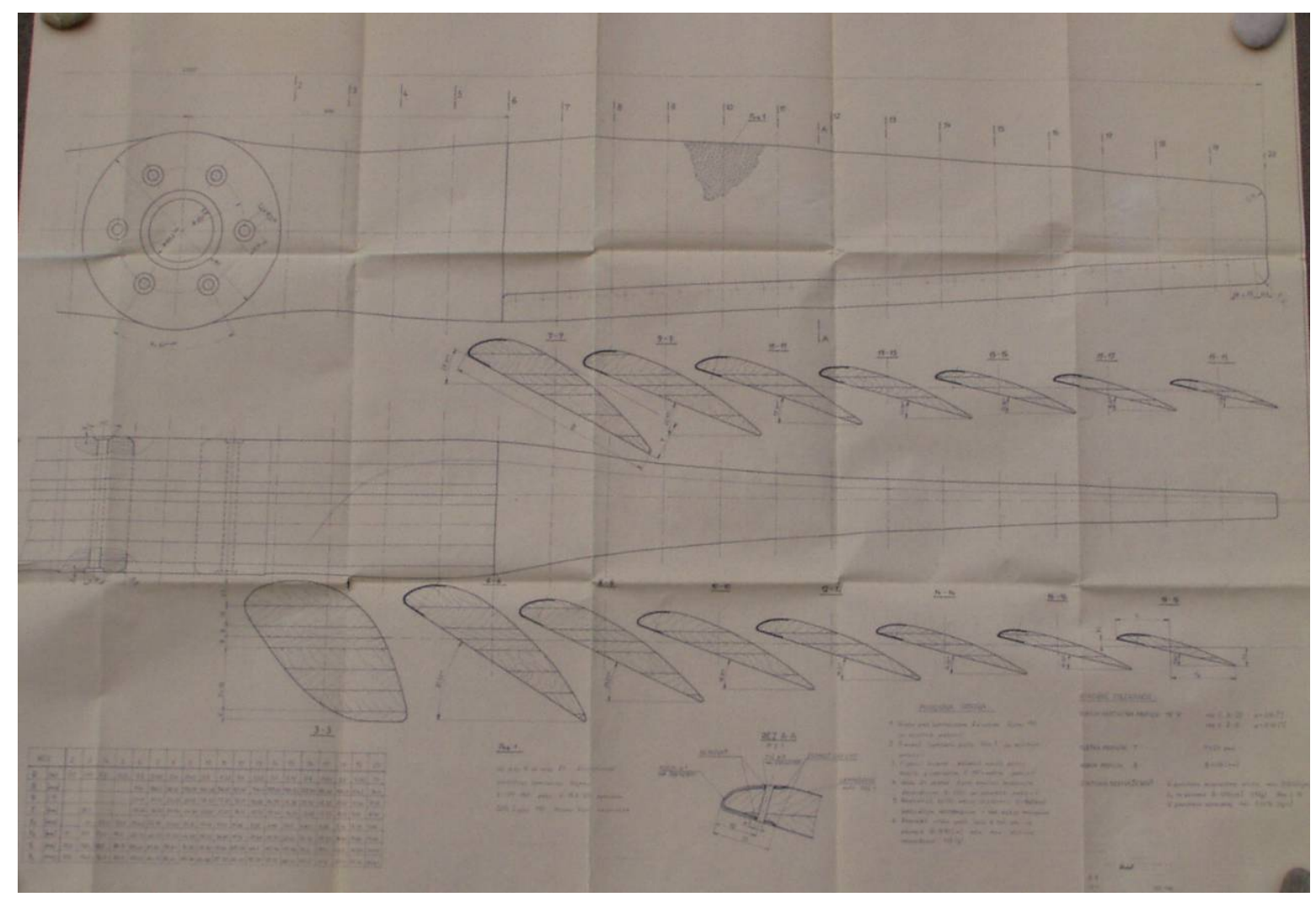

Fig.5. Propeller lay-out (Prop. SC-Z226)

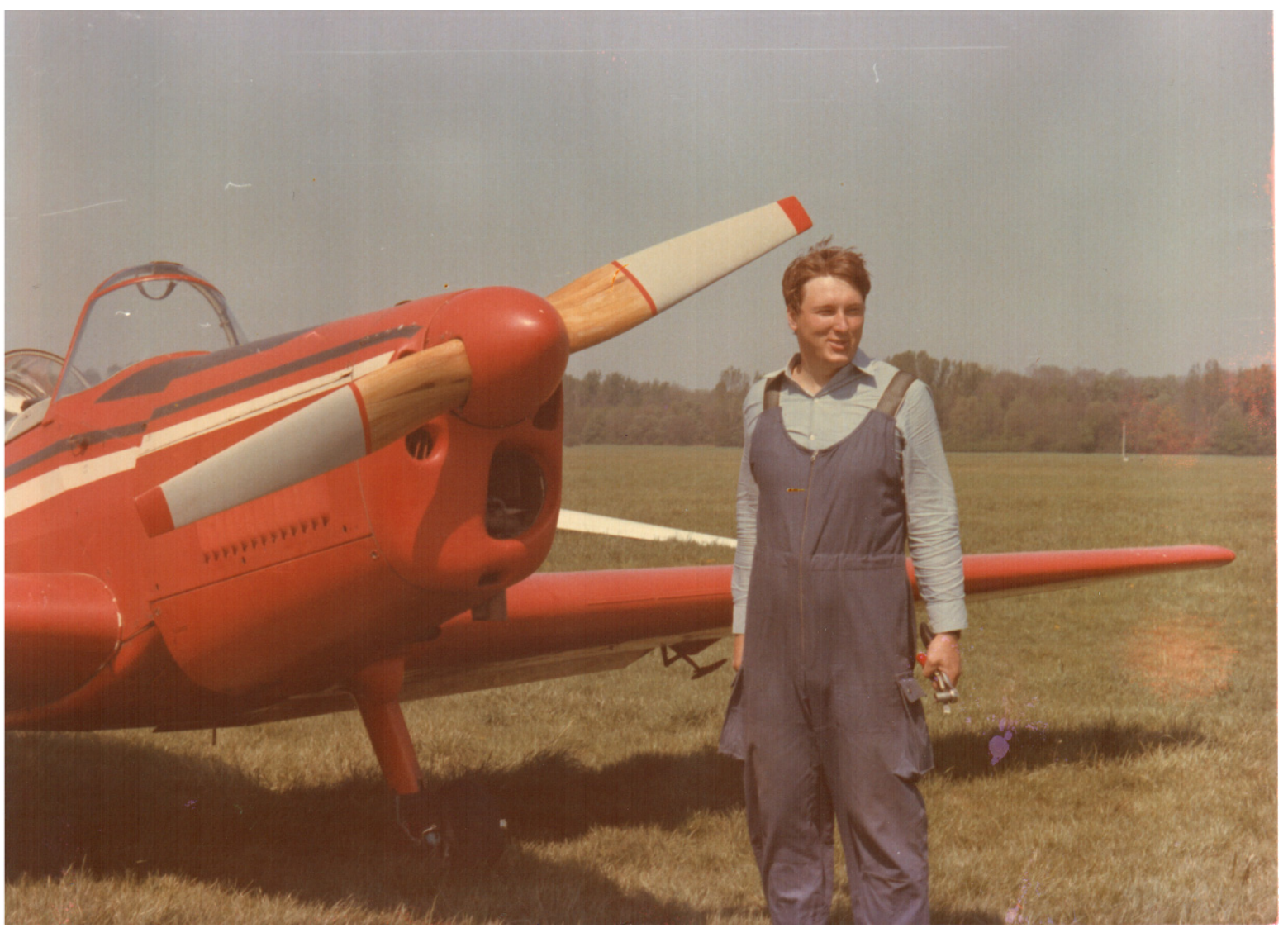

Fig.6. Realization of the propeller SC-Z226

$10 / 10$ 